\title{
HER2 testing in gastric cancer: a practical approach
}

Josef Rüschoff ${ }^{1,2}$, Wedad Hanna ${ }^{3}$, Michael Bilous ${ }^{4}$, Manfred Hofmann², Robert Y Osamura ${ }^{5}$, Frédérique Penault-Llorca ${ }^{6}$, Marc van de Vijver ${ }^{7}$ and Giuseppe Viale ${ }^{8}$

${ }^{1}$ Targos Molecular Pathology GmbH, Kassel, Germany; ${ }^{2}$ Institute of Pathology Nordhessen, Kassel, Germany; ${ }^{3}$ Department of Laboratory Medicine and Pathobiology, Anatomic Pathology, Sunnybrook Health Sciences Center, Faculty of Medicine, University of Toronto, Toronto, Canada; ${ }^{4}$ Healthscope Pathology, Norwest Private Hospital, Faculty of Medicine University of Sydney, Sydney, Australia; ${ }^{5}$ International University of Health and Welfare (IUHW), Center for Diagnostic Pathology, IUHW Mita Hospital, Tokyo, Japan; ${ }^{6}$ Department of Pathology, Centre Jean Perrin and University of Auvergne, Clermont-Ferrand, France; ${ }^{7}$ Department of Pathology, The Academic Medical Center (AMC), The University of Amsterdam, Amsterdam, The Netherlands and ${ }^{8}$ Department of Pathology and Laboratory Medicine, University of Milan and European Institute of Oncology, Milan, Italy

Trastuzumab in combination with capecitabine or 5 -fluorouracil and cisplatin is approved by the European Medicines Agency for the treatment of patients with human epidermal growth factor receptor 2 (HER2)-positive (immunohistochemistry $3+$ or immunohistochemistry $2+$ /fluorescence in situ hybridization-positive or immunohistochemistry $2+$ /silver in situ hybridization-positive) metastatic adenocarcinoma of the stomach or gastro-esophageal junction. Approvals are underway in other countries, with recent approvals granted in the United States and Japan. Experience and data from trastuzumab use in breast cancer have highlighted the importance of quality HER2 testing and scoring to ensure accurate identification of patients eligible for treatment. HER2 testing in gastric cancer differs from testing in breast cancer due to inherent differences in tumor biology; gastric cancer more frequently shows HER2 heterogeneity (focal staining) and incomplete membrane staining. Consequently, gastric cancer-specific HER2 testing protocols have been developed and standardized and it is imperative that these recommendations be adhered to. Given the predictive value of HER2 protein levels with response in the trastuzumab for GAstric cancer study (ToGA), immunohistochemistry should be the initial testing methodology and fluorescence in situ hybridization or silver in situ hybridization should be used to retest immunohistochemistry $2+$ samples. Wherever possible, bright-field methodologies should be used as these are considered to be superior to fluorescent methodologies at identifying heterogeneous staining. Specific training is required before embarking on HER2 testing in gastric cancer, irrespective of the experience of HER2 testing in breast cancer. This paper provides the most up-to-date practical guidance on HER2 testing and scoring in patients with gastric and gastro-esophageal junction cancer, as agreed by a panel of expert pathologists with extensive experience of HER2 testing particularly reflecting the European Medicines Agencyapproved indication. It is anticipated that these recommendations should ensure accurate and consistent HER2 testing, which will allow appropriate selection of patients eligible for treatment with trastuzumab.

Modern Pathology (2012) 25, 637-650; doi:10.1038/modpathol.2011.198; published online 6 January 2012

Keywords: gastric cancer; guidance; HER2 testing; immunohistochemistry; in situ hybridization; quality assurance; trastuzumab

In July 2010, a panel of expert pathologists from the European Union and the rest of the world, with

Correspondence: Professor J Rüschoff, Targos Molecular Pathology $\mathrm{GmbH}$, Germaniastr 7, 34119 Kassel, Germany.

E-mail: rueschoff@patho-nordhessen.de

Received 22 December 2010; revised 5 October 2011; accepted 5

October 2011; published online 6 January 2012 extensive experience of human epidermal growth factor receptor 2 (HER2) testing, met to discuss the requirements for accurate HER2 testing and interpretation in gastric cancer. The recommendations generated from this meeting are summarized in this paper and are based on both the key findings from the trastuzumab for GAstric cancer study ${ }^{1}$ and the expertise of the authors in HER2 testing for both breast and gastric cancers. ${ }^{2-7}$ 
The objective of this paper is to provide up-to-date guidance on standardizing tissue processing, HER2 testing, and scoring in patients with gastric and gastro-esophageal junction cancer to help ensure accurate selection of patients eligible for treatment with trastuzumab. These recommendations are intended not only to complement existing recommendations but also to provide more practical guidance on HER2 testing specific for gastric cancer. When recommendations are made in this article they are made by the panel, unless otherwise stated. It is anticipated that the recommendations will evolve over time as more information on HER2 testing in gastric cancer becomes available.

\section{Epidemiology}

Gastric cancer is associated with substantial morbidity and mortality worldwide. It is the fourth most commonly diagnosed cancer and the second most common cause of cancer-related deaths globally. ${ }^{8-10}$ The epidemiology of metastatic gastric cancer varies across geographical areas, ${ }^{8,9}$ and while the incidence of metastatic gastric cancer is low in countries such as Japan where screening programs identify gastric cancer at early stages, ${ }^{11}$ in Western countries without screening programs, late diagnosis is common and a particular challenge. The majority of patients with gastric cancer present with advanced, metastatic, or inoperable disease, and have a poor prognosis. Although screening in Japan has led to a decrease in death rates for gastric cancer (twofold decrease in screened vs unscreened subjects), improved 5-year survival rates, and a significant reduction in the overall incidence of advanced gastric cancer ${ }^{11,12}$ in general, the median 5-year survival rates reported for metastatic gastric cancer are in the range of $5-20 \%$ in the Western populations..$^{9,13,14}$

\section{HER2 as a therapeutic target in gastric cancer}

HER2 is a key driver of tumorigenesis, and overexpression as a result of HER2 gene amplification has been observed in a number of solid tumors. ${ }^{15,16}$ HER2 is a well-established therapeutic target in breast cancer, and while therapies that target HER2 are now considered the standard of care in this indication, ${ }^{17-20}$ it has become clear that a tailored approach to patient selection is required. There is increasing evidence that HER2 is an important biomarker in gastric and gastro-esophageal junction tumors; ${ }^{21}$ analysis of HER2 status by immunohistochemistry and in situ hybridization techniques, using different scoring methods or assays, suggests that HER2 is overexpressed in $\sim 7-34 \%$ of gastric tumors. ${ }^{7,21-26}$ Results from the international, randomized, Phase III trastuzumab for GAstric cancer study demonstrated a survival benefit with trastuzumab plus chemotherapy (capecitabine or 5-fluorouracil and cisplatin) in patients with HER2-positive locally advanced, recurrent and/or metastatic gastric or gastro-esophageal junction tumors that overexpress HER2. ${ }^{1}$ Patients with high HER2-expressing tumors derived the greatest benefit from trastuzumab therapy. HER2 positivity as defined in the trastuzumab for GAstric cancer study was immunohistochemistry $3+$ and/or fluorescence in situ hybridizationpositive (HER2:chromosome 17 ratio $\geq 2.0$ ).

On the basis of these findings, it is now recommended that all patients with gastric cancer should have their tumors tested for HER2 status at the time of initial diagnosis. According to the European Medicines Agency license, patients with HER2-positive metastatic disease whose tumors are immunohistochemistry $3+$ or immunohistochemistry $2+$ /fluorescence in situ hybridization-positive or immunohistochemistry $2+$ /silver in situ hybridization-positive are eligible for trastuzumab therapy. ${ }^{27}$ Regulatory approval for trastuzumab was granted in October 2010 in the United States for patients with metastatic adenocarcinoma of the stomach or gastro-esophageal junction whose tumors were HER2-positive as determined using approved testing methods, and in Japan in 2011 for patients with inoperable advanced or recurrent HER2-overexpressing disease. As the expert panel met before American and Japanese approval, American- and Japan-specific recommendations are outside the scope of this paper.

Given that the majority of patients present with advanced disease and that gastric cancer is a very rapidly progressing cancer, it is highly recommended that the turnaround time from the histological diagnosis of gastric cancer to reporting of the HER2 result should not exceed 5 working days. To achieve such fast turnaround times and to maintain high-quality HER2 testing it is important to adopt a multidisciplinary approach, with testing performed in laboratories with training and experience in HER2 testing for gastric cancer.

\section{Unique features of HER2 testing in gastric cancer}

The extensive experience of HER2 testing in breast cancer has highlighted the importance of optimizing HER2 testing and interpretation to ensure that patients who may derive benefit receive appropriate targeted therapy. Gastric cancer exhibits unique immunostaining characteristics compared with breast cancer, including the high incidence of tumor heterogeneity (defined as $<30 \%$ of tumor cells staining positive or only focal staining of tumor cells) in up to $30 \%$ of HER2-positive cases. ${ }^{7,28}$ Another key difference from breast cancer staining is that HER2-positive gastric carcinomas are usually of the gland-forming intestinal type and may show incomplete, basolateral, or lateral staining in 
addition to complete membrane staining and all these are considered as a positive result with immunohistochemistry (Figure 1b). These differences have been taken into account when devising the optimal HER2 testing protocol for gastric cancer and the protocols have been further developed, standardized, and refined in testing and, posttrastuzumab for GAstric cancer, further recommendations have been proposed to ensure quality HER2 testing based on testing data and expert opinion. ${ }^{1,7,29}$

Data from the trastuzumab for GAstric cancer study demonstrated that patients with tumors that had high levels of HER2 protein expression (immunohistochemistry $3+$ or immunohistochemistry $2+$ /fluorescence in situ hybridizationpositive) derived the greatest benefit from treatment with trastuzumab ${ }^{1}$ and consequently immunohistochemistry should be the initial testing method; fluorescence in situ hybridization or silver in situ hybridization should be used to retest immuno- histochemistry $2+$ samples. It is our recommendation that, wherever possible, bright-field methodologies are preferred to help to identify regions of heterogeneous staining.

In order to ensure accurate and reproducible HER2 testing results, it is essential that interpretation of HER2 results is performed with strict adherence to the scoring criteria specific for gastric cancer as reported in the trastuzumab for GAstric cancer study and the recommendations of the expert panel; scoring criteria have been developed and published (Table 1). ${ }^{1}$ Applying the breast cancer testing principles and scoring criteria to HER2 scoring in patients with gastric cancer may result in the underscoring of tumors, and recent evidence suggests that the rate of false-negatives observed may be as high as $50 \%$ compared with the trastuzumab for GAstric cancer trial, thus preventing eligible patients having access to trastuzumab therapy. ${ }^{29}$ See Table 2 for a comparison of breast and
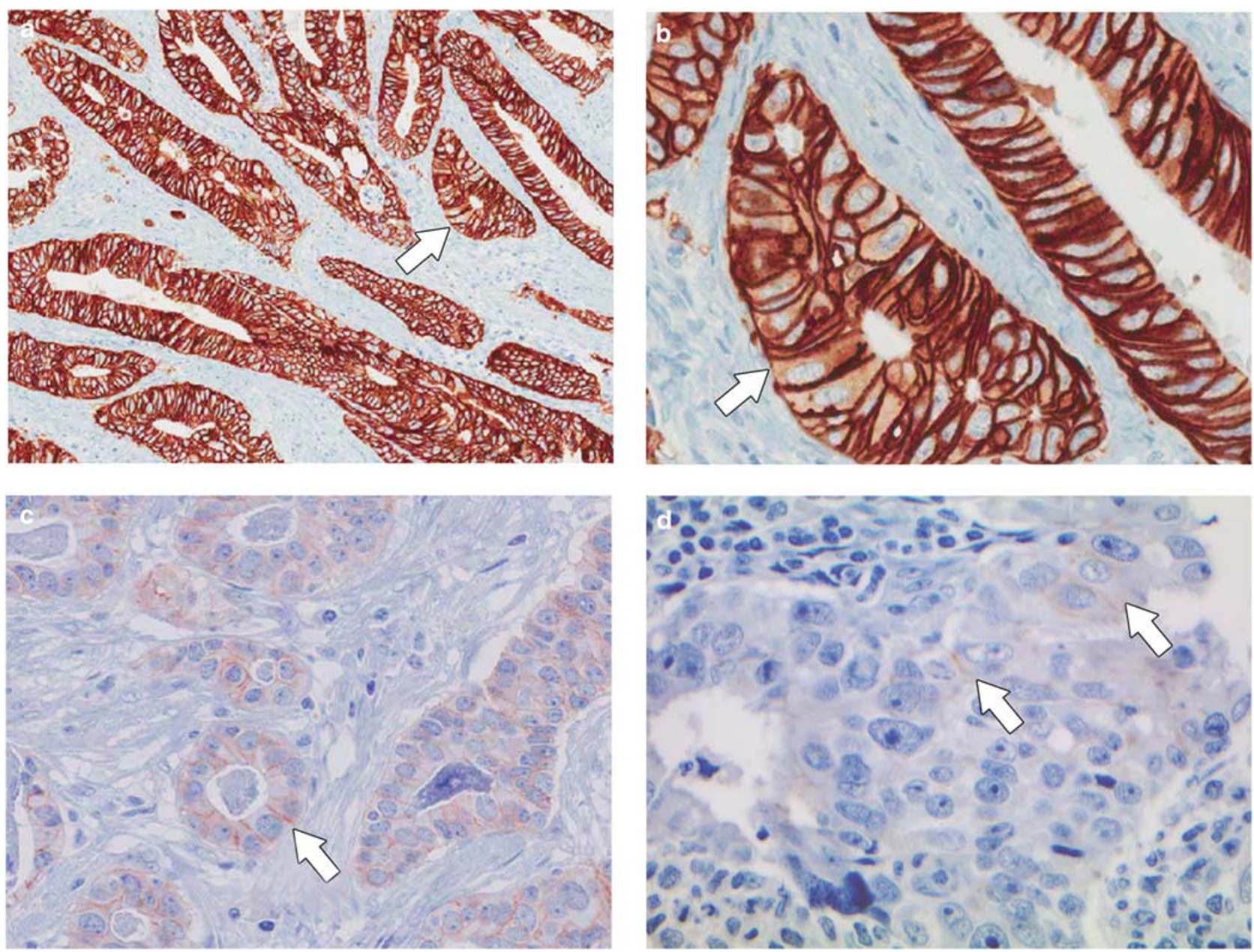

Figure 1 (a-d) HER2 immunoscoring according to the 'magnification rule.' (a) Immunohistochemistry $3+$ case with distinct membranous staining discernible at low magnification $(\times 5$; see arrow) with (b) lateral- or U-shaped membranous staining typical at the cell-cell junctions $(\times 40$, see arrow). (c) Immunohistochemistry $2+$ case with membranous staining unequivocally visible only if a medium magnification is used $(\times 20$; see arrow). (d) Immunohistochemistry $1+$ staining corresponds to membranous staining that is clearly visible only at high $\times 40$ magnification (see arrow). 
Table 1 Human epidermal growth factor receptor 2 (HER2) scoring criteria for gastric cancer

\begin{tabular}{|c|c|c|c|}
\hline Score & Surgical specimen-staining pattern & Biopsy specimen-staining pattern & $\begin{array}{l}\text { HER2 overexpression } \\
\text { assessment }\end{array}$ \\
\hline 0 & $\begin{array}{l}\text { No reactivity or membranous reactivity in } \\
<10 \% \text { of tumor cells }\end{array}$ & $\begin{array}{l}\text { No reactivity or no membranous reactivity in any } \\
\text { tumor cell }\end{array}$ & Negative \\
\hline $1+$ & $\begin{array}{l}\text { Faint/barely perceptible membranous } \\
\text { reactivity in } \geq 10 \% \text { of tumor cells; cells } \\
\text { are reactive only in part of their membrane }\end{array}$ & $\begin{array}{l}\text { Tumor cell cluster with a faint/barely perceptible } \\
\text { membranous reactivity irrespective of percentage } \\
\text { of tumor cells stained }\end{array}$ & Negative \\
\hline $2+$ & $\begin{array}{l}\text { Weak to moderate complete, basolateral, } \\
\text { or lateral membranous reactivity in } \geq 10 \% \\
\text { of tumor cells }\end{array}$ & $\begin{array}{l}\text { Tumor cell cluster with a weak to moderate complete, } \\
\text { basolateral, or lateral membranous reactivity } \\
\text { irrespective of percentage of tumor cells stained }\end{array}$ & Equivocal \\
\hline $3+$ & $\begin{array}{l}\text { Strong complete, basolateral, or lateral } \\
\text { membranous reactivity in } \geq 10 \% \text { of } \\
\text { tumor cells }\end{array}$ & $\begin{array}{l}\text { Tumor cell cluster with a strong complete, basolateral, } \\
\text { or lateral membranous reactivity irrespective of } \\
\text { percentage of tumor cells stained }\end{array}$ & Positive \\
\hline
\end{tabular}

Table 2 Comparison of differences between human epidermal growth factor receptor 2 (HER2) scoring in gastric and breast cancer ${ }^{29}$

\begin{tabular}{|c|c|c|c|}
\hline & & Gastric cancer & Breast cancer \\
\hline \multirow{3}{*}{$\begin{array}{l}\text { Immunohistochemical } \\
\text { scoring }\end{array}$} & Extent & Biopsy specimens $\geq 5$ cells & $\geq 10 \%(\geq 30 \%)^{\mathrm{a}}$ \\
\hline & (Area cutoff) & Resection specimens: $\geq 10 \%$ & \\
\hline & Circularity & $\begin{array}{l}\text { Mostly missing (often only lateral } \\
\text { in immunohistochemistry } 2+/ 3+\text { ) }\end{array}$ & $\begin{array}{l}\text { A must in immuno- } \\
\text { histochemistry } 2+/ 3+\end{array}$ \\
\hline \multirow[t]{2}{*}{$\begin{array}{l}\text { (Fluorescence) in situ } \\
\text { hybridization analysis }\end{array}$} & Cell number & $\begin{array}{l}20 \text { cohesive tumor cells } \\
\text { showing highest gene count } \\
\text { (add } 20 \text { new if ratio } 1.8-2.2 \text { ) }\end{array}$ & $\begin{array}{l}20 \text { cohesive tumor cells } \\
\text { showing highest gene count } \\
\text { (add } 20 \text { new if ratio } 1.8-2.2 \text { ) }\end{array}$ \\
\hline & Amplification & Ratio $\geq 2.0$ & Ratio $\geq 2.0(\geq 2.2)^{\mathrm{a}}$ \\
\hline \multirow[t]{2}{*}{ HER2 positivity } & Tumor type & $\begin{array}{l}\text { About } 30 \% \text { of intestinal type gastric } \\
\text { cancer about } 15 \% \text { of mixed type } \\
\text { about } 5 \% \text { of diffuse type (signet } \\
\text { ring type typically negative) }\end{array}$ & $\begin{array}{l}\text { 15-25\% of ductal type } \\
\text { (grade } 2 / 3 \text { ); almost never } \\
\text { in subtypes such as lobular, } \\
\text { medullary, and ductal grade } 1\end{array}$ \\
\hline & Tumor location & $\begin{array}{l}\text { About } 30 \% \text { at cardiac/gastro- } \\
\text { esophageal junction About } 15 \% \\
\text { of gastric cancer }\end{array}$ & No correlation \\
\hline Patient selection & $\begin{array}{l}\text { Fluorescence in situ hybridi- } \\
\text { zation vs immunohistochemistry }\end{array}$ & $\begin{array}{l}\text { Immunohistochemistry more } \\
\text { predictive than fluorescence } \\
\text { in situ hybridization: immuno- } \\
\text { histochemistry primary test } \mathrm{t}^{\mathrm{b}} \\
\text { fluorescence in situ hybridization } \\
\text { only if immunohistochemistry 2+ }\end{array}$ & $\begin{array}{l}\text { Fluorescence in situ } \\
\text { hybridization/immuno- } \\
\text { histochemistry equally } \\
\text { predictive }\end{array}$ \\
\hline
\end{tabular}

\footnotetext{
${ }^{\mathrm{a}}$ According to the American Society of Clinical Oncology/College of American Pathologists. ${ }^{30}$

${ }^{\mathrm{b}}$ According to approval by the European Medicines Agency. ${ }^{27}$
}

gastric cancer scoring according to the American Society of Clinical Oncology/College of American Pathologists $^{30}$ and the European Medicines Agency $^{27}$ recommendations.

\section{Practical guidance on HER2 testing in gastric cancer}

The following sections will provide the most upto-date practical guidance on how to conduct HER2 testing in gastric cancer, from initial sample collection through to scoring the results, and how to ensure quality testing through quality assurance schemes.

\section{Sample Collection}

Although the American Society of Clinical Oncology/College of American Pathologists recommendations for breast cancer ${ }^{30}$ form a solid basis for HER2 testing in gastric cancer, modifications are required to take into account the unique characteristics of this tumor type.

Both surgical specimens and biopsy samples are acceptable for HER2 testing in gastric cancer and the availability of specimen type may vary according to region: for example, surgical specimens are more common in Japan (although biopsies are also available). On the basis of more standardized fixation conditions, it is generally agreed that 
biopsies are preferred to ensure optimal testing results. A representative block of the cancer, including the intestinal tumor part, should be taken from surgical specimens and when biopsies are taken, adequate numbers of viable endoscopic biopsies (ideally six to eight) of the cancer are recommended for HER2 testing to reflect possible heterogeneity of the tumor. ${ }^{31,32}$ In countries where it is common for only one or two biopsies to be collected, all available samples should be tested for HER2 status. Tissue microarrays are not suitable for HER2 testing to inform clinical decision making due to the heterogeneous nature of HER2 overexpression and amplification in this tumor type. ${ }^{3}$ As stated above, it is important to ensure that sufficient tumor biopsy specimens are collected as only some of the selected biopsies may show HER2 positivity due to tumor heterogeneity (Figure 2a). Furthermore, many biopsy specimens are found to be necrotic in nature or shown not to contain tumor tissue, and specimens are often affected by sample preparation artifacts (edge artifacts and crushing artifacts), making them unsuitable for assessment. ${ }^{29}$ For metastatic cases, performing a second biopsy at the site of metastasis may also be informative; however, unlike in breast cancer cases, there are currently no valid data available on concordance between HER2 status in primary tumors and derived metastases in gastric cancer.

The time from biopsy or excision to placing in formalin fixative can affect the HER2 testing result and it is recommended that once tissue has been
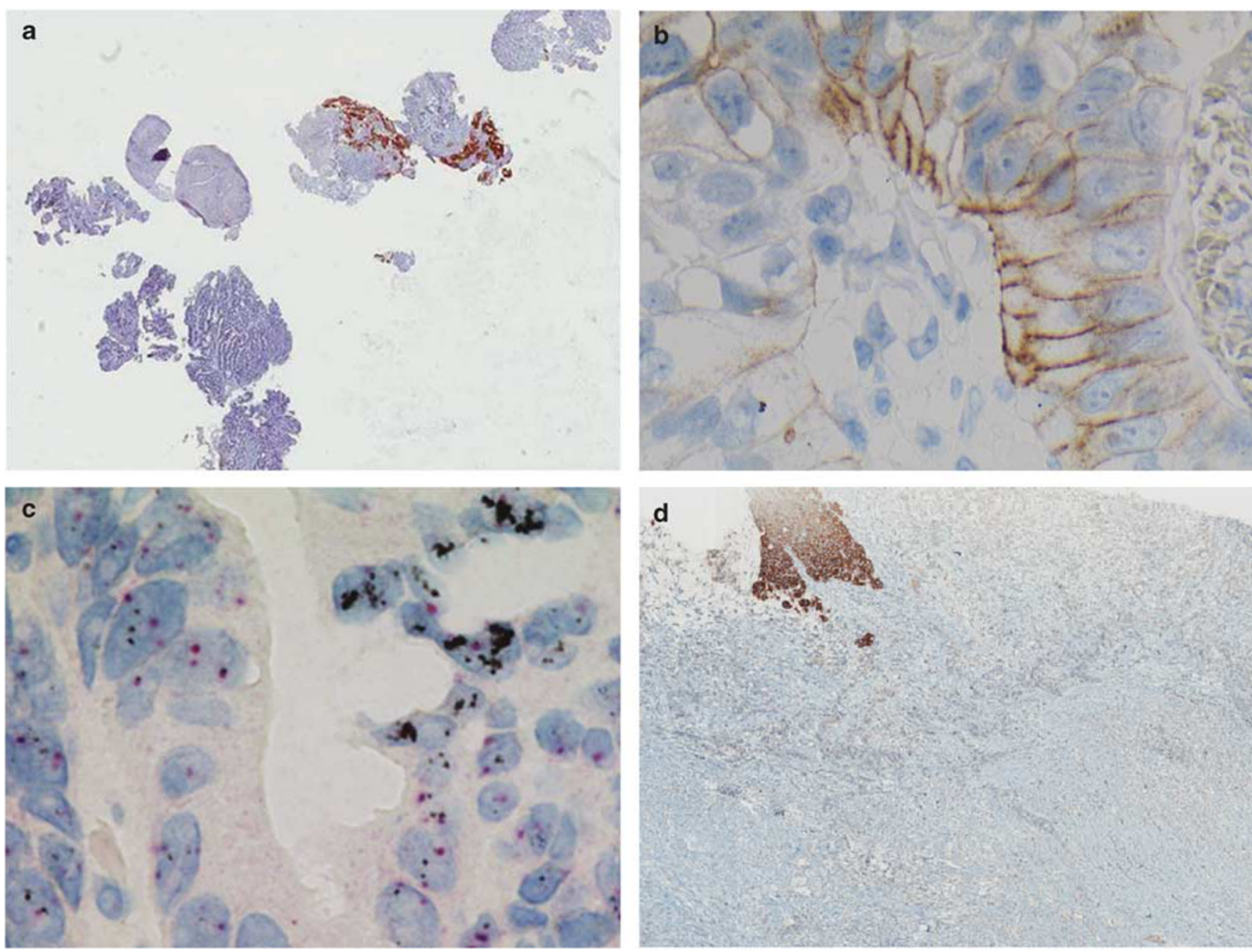

Figure 2 (a-g) HER2 heterogeneity and polysomy in gastric cancer. (a-c) Biopsy material comprising around eight samples endoscopically removed. (a) Strong HER2 expression in only two of the biopsy samples (upper right) corresponding to immunohistochemistry $3+$ (strong membranous staining visible in a group of $\geq 5$ cells at $\times 2.5$ ). (b) Other areas of this tumor show heterogeneous immunohistochemistry $2+$ expression with only some focally stained tumor cells within a gland of this intestinal-type adenocarcinoma $(\times 40)$. (c) The same tumor cells exhibit focal HER2 gene amplification (dual color bright-field in situ hybridization, $\times 40)$. (d-f) Heterogeneous HER2 expression in a resection specimen of a mixed-type adenocarcinoma. (d) A solid growing nest of cells with residual poorly differentiated carcinoma $(\times 4)$ with $(\mathbf{e})$ intense, clearly membranous HER2 expression $<10 \%$ of the tumor $(\sim 1 \%$; $\times 40)$; this case was scored as immunohistochemistry 0 . (f) In situ hybridization testing revealed high HER2 amplification $(\sim 200$ cells; $\times 40)$, which according to testing recommendations corresponds to a HER2-positive result. (g) Polysomy in a gastric cancer specimen. The mean chromosome 17 count was 4.5 red signals/tumor cell and mean HER2 gene count was eight black signals/tumor cell $(\times 63)$. Although the HER2:chromosome ratio was $<2.0$ (1.78), this case is regarded as HER2-positive due to gene count $>6$. 

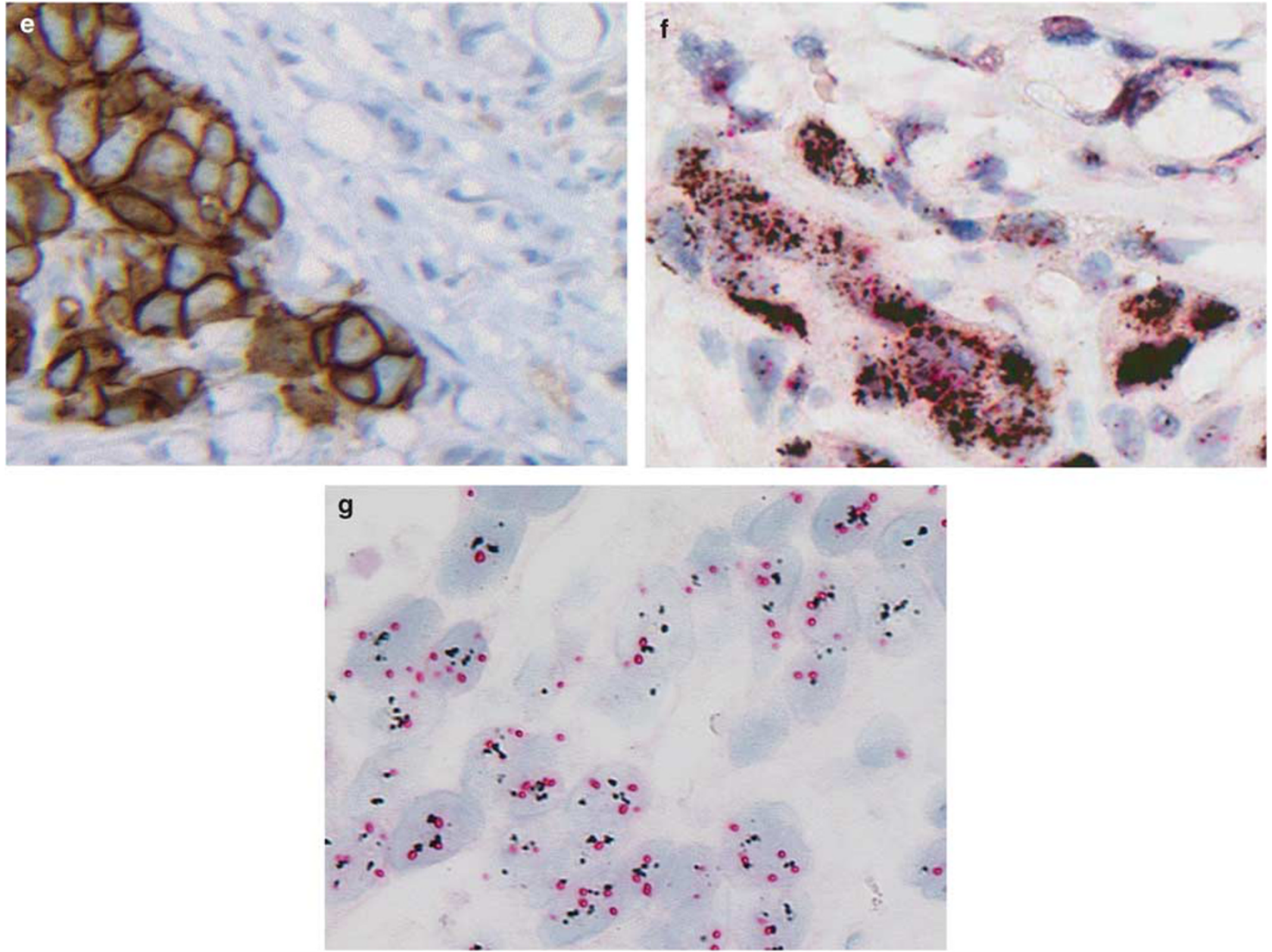

Figure 2 Continued.

acquired, it should be fixed immediately (especially important for biopsies as they can dry very quickly) and within $20 \mathrm{~min}$ of excision in the case of surgical specimens, if possible. Fixation should be performed in the operating theater if the sample cannot be transported to the laboratory promptly. Excision specimens should be inked and fixed (resection specimens should be opened and pinned on a corkboard before fixation); sutures and/or clips can be used to orientate the specimen. The date and time of specimen acquisition should always be recorded.

\section{Tissue Preparation}

Standardization of tissue preparation should take into account each stage of the process, including sample collection, fixation, paraffin embedding, sectioning, deparaffinization of sections, and immunohistochemical/in situ hybridization testing.

Poor fixation is a common and important source of error in HER2 testing ${ }^{30,33}$ and to ensure adequate fixation for all gastric cancer specimens, the preferred fixative is $10 \%$ neutral-buffered formalin (equivalent to $4 \%$ formaldehyde solution). Fresh formalin should be used wherever possible; the use of any alternative fixatives is not recommended, as this requires prior validation. For both surgical and biopsy specimens the fixation time in formalin should be a minimum of $8 \mathrm{~h}$ and a maximum of $48 \mathrm{~h}$. Fixation time is of particular importance when biopsy specimens are processed by rapid embedding with a shortened formalin fixation time as this can lead to false-positive immunohistochemical HER2 staining. Therefore, fixative type and fixation duration should always be documented and where there is any doubt quality assurance measures should be taken (see section 'Quality Assurance').

Following fixation, tissue samples should be dehydrated in an ethanol/xylene series and embedded using fresh paraffin wax maintained at $55-60{ }^{\circ} \mathrm{C}$. Exposure of samples to higher temperatures may degrade epitopes so prolonged incubation in molten paraffin wax should be avoided. Once embedded in paraffin wax, samples can be stored at room temperature $\left(20-25^{\circ} \mathrm{C}\right)$, before sectioning.

It is recommended that sections should be cut from the tissue block immediately before testing to preserve antigenicity. Sections must be cut from 
a representative area of the gastric or gastro-esophageal junction tumor (eg, the region containing adenocarcinoma of intestinal type in the case of mixed cancer) and from viable regions of the tumor. Histology should be confirmed with a hematoxylin and eosin section. The standard thickness of gastric cancer sections should be $\sim 4 \mu \mathrm{m}$ or less to ensure accurate visualization and interpretation of assay results. Sections should be mounted on slides with good adhesion properties (eg, Superfrost slides or poly-L-lysine-coated slides) and dried for $12-24 \mathrm{~h}$ at room temperature or $1 \mathrm{~h} 60^{\circ} \mathrm{C}$. Before HER2 testing with either immunohistochemistry or in situ hybridization, sections are deparaffinized and rehydrated in a xylene (or xylene substitute)/ethanol series at room temperature. It is important to ensure complete removal of paraffin wax during this process as failure to do so can reduce specific staining and increase nonspecific background staining in immunohistochemistry.

\section{Optimizing Immunohistochemistry}

It is highly recommended that a standard operating procedure for processing gastric tissue be developed within each testing laboratory, including standardization of tissue processing protocols and laboratory equipment.

One important recommendation is that validated immunohistochemical testing methodologies are used to ensure accuracy and consistency of HER2 testing. Currently, two validated immunohistochemical testing kits are commercially available for HER2 analysis in gastric cancer (availability varies according to regional regulations) and these are the Ventana anti-HER-2/neu (4B5) Rabbit Monoclonal Primary Antibody kit (Ventana/Roche Tissue Diagnostics) and the HercepTest (Dako). Validated kits will generate more accurate, reliable results than in-house kits and the manufacturer's instructions should always be followed. Initial data on proficiency testing within the Quality assurance Initiative in Pathology study in Germany have demonstrated that the choice of testing kit or use of an in-house methodology can have a significant impact on reliability and reproducibility of results (Baretton G, 2010; personal communication). Another key suggestion for optimizing immunohistochemistry is that sections mounted on slides should be stained within 2-4 weeks of sectioning in order to maintain antigenicity and ensure quality immunohistochemical HER2 testing results are achieved.

An antigen retrieval step is required before immunohistochemical staining of gastric cancer tissue, as antigenicity may be masked by the fixation process. The extent of antigen retrieval required is dependent on tissue fixation and primary antibody and it is strongly advised that automated, temperature-controlled antigen retrieval and staining equipment be used, as antigen retrieval is a frequent source of variation in HER2 staining results. Inappropriate antigen retrieval methods can activate endogenous biotin, leading to high levels of background staining with avidin-biotin reaction-based detection. ${ }^{34}$

Whenever immunohistochemistry is performed for HER2, it is essential that both positive and negative controls are included in each run. When possible, on-slide controls with defined HER2 expression levels should be included on each sample slide and if available, cell lines with defined HER2 expression levels can also be used as run-controls. Controls should be prepared using similar fixation- and paraffin-embedding methods to the test samples. The controls provided or recommended in the validated kits for gastric cancer should be used wherever possible. For practical purposes tissue microarrays comprising tumor cores of different HER2 status may be valuable tools for quality control assessments. In particular, outside of high-throughput labs, tissue microarrays can provide a rapid overview of testing quality. Tissue microarrays need to be carefully constructed to avoid bias by tumor heterogeneity as otherwise they may not be appropriate for quality assurance purposes.

\section{Sample Analysis: Immunohistochemistry}

When interpreting HER2 staining, it is important to be aware of focal staining that is commonly reported in gastric cancer ${ }^{7,28,29}$ and the finding that gastric tumor cells often show incomplete HER2 membrane reactivity that can be basolateral or lateral in distribution (Figure 1b). One key feature of the gastric cancer-specific scoring criteria is the inclusion of strong incomplete membrane staining as HER2-positive if $\geq 10 \%$ cells or $\geq 5$ clustered cells are stained for surgical and biopsy samples, respectively. ${ }^{1,29}$ Therefore, the overall result is based on the highest positive scoring sample without averaging the results in multiple biopsy specimens. The cutoff of $10 \%$ of cells was applied as part of the United States Food and Drug Administration approval, based on the results of the trastuzumab for GAstric cancer trial in surgical excision specimens. However, due to the marked heterogeneity of HER2 found in the pre-trastuzumab for GAstric cancer study $^{7}$ the $10 \%$ cutoff was disregarded for biopsy specimens. In the trastuzumab for GAstric cancer study, the lowest number of tumor cells scored in biopsy samples was $\sim 200$ (Targos data on file). A German-French ring study provided further data to support the use of a cluster of $\geq 5$ tumor cells, and showed the highest level of concordance between participants. $^{29}$ As the majority of HER2-overexpressing cells (immunohistochemistry $3+$ ) also show gene amplification, the same number of cells (ie, 20) should be counted for both techniques to be evaluated. Only cells showing specific membrane 
staining at the cell-to-cell contact sites (not at the luminal or apical parts) should be evaluated. This excludes single, noncohesive stained cells from scoring, as these cells often correspond to nonspecifically stained signet ring cells (which typically show no HER2 gene amplification). Accordingly the panel recommends that if immunohistochemistry is used as the initial test, any specimen type (either surgical excision or biopsies) with $<10 \%$ strongly stained tumor cells should be subjected to confirmatory in situ hybridization testing to preclude false-negative results, and that any case that shows a positive result using one of the test platforms, regardless of the specimen type, should be considered HER2-positive.

Samples with poorly preserved tissue (including edge crushing or shrinkage artifacts), nonspecific staining (eg, cytoplasmic or nucleic staining), or staining in the normal mucosa (particularly intestinal metaplasia) should be excluded or retested with fluorescence in situ hybridization or silver in situ hybridization (Figures 3a-d).

In addition to these formal scoring criteria, it has been shown that determining the microscopic magnification at which specific membranous staining is visible overcomes the problem of visual illusions associated with brightness perception in general (see: Checker Shadow Illusion by Adelson EH, Cambridge; http://web.mit.edu/persci/people/ adelson/checkershadow_illusion.html). Accordingly, when the observer concentrates not only on the intensity of staining, but also focuses on the identification of a distinct structure (ie, membranous staining), interobserver concordance is markedly increased. ${ }^{29}$ Therefore, the 'magnification rule' should always be used in conjunction with the gastric cancer-specific scoring criteria. ${ }^{29}$ Considering the degree of microscopic magnification required to identify membranous staining is helpful in determining the immunohistochemistry score, particularly in borderline (eg, immunohistochemistry $2+v S$ immunohistochemistry 1+) and difficult (eg, heterogeneous and focally stained) cases. Briefly, according to the 'magnification rule':

- Immunohistochemistry $3+$ staining is defined as any membranous staining visible at low magnification ( $\times 2.5-5$; Figure 1a). Lateral- or U-shaped membranous staining is typically seen at cell-cell junctions (Figure 1b).

- Immunohistochemistry $2+$ membranous staining is visible at $\times 10-20$ magnification (Figure 1c). Immunohistochemistry $2+$ cases should be retested using fluorescence in situ hybridization or silver in situ hybridization.

- Immunohistochemistry $1+$ staining is visible only with $\times 40$ magnification and should be considered immunohistochemistry-negative (Figure 1d).

When the Ventana anti-HER-2/neu (4B5) Rabbit Monoclonal Primary Antibody kit (Roche Tissue Diagnostics) and the Ventana HER2 DNA probe (formerly known as BDISH; Roche Tissue Diagnostics) were used in conjunction with the 'magnification rule,' good concordance between immunohistochemistry and in situ hybridization was observed. ${ }^{29}$ In a series of 152 diagnostic gastric cancer specimens, all immunohistochemistry $3+$ tumors $(n=24)$ also showed HER2 gene amplification (in situ hybridization-positive). Polysomy and low-level amplification was restricted to the $32 \%$ of in situ hybridizationpositive/immunohistochemistry $2+\quad(n=47)$ cases and the $5 \%$ of in situ hybridization-positive/immunohistochemistry $1+(n=41)$ tumors. ${ }^{29}$

\section{Interpreting and Retesting Borderline and Difficult Immunohistochemistry Cases}

Inevitably there will be cases that cannot be easily interpreted using the recommendations defined in Section 'Sample Analysis: Immunohistochemistry'. These cases include borderline $1+/ 2+$ staining seen by immunohistochemistry, the presence of artifacts including 'edge effects,' and nonspecific cytoplasmic or normal cell staining. Currently, there is uncertainty surrounding the responsiveness of patients with immunohistochemistry 0/immunohistochemistry $1+$ tumors to trastuzumab and this is reflected in the different country-specific regulations. It is recommended that for these borderline cases and for other 'problematic' cases, retesting should be performed with fluorescence in situ hybridization or silver in situ hybridization; if the fluorescence in situ hybridization or silver in situ hybridization result is positive then the tumor can be scored as HER2-positive. The scores for both assays should be indicated separately on the report. It is also appropriate to score an alternative sample of the tumor or a lymph node containing a metastasis in these cases.

Furthermore, given the heterogeneous nature of HER2 staining in gastric cancer, it is suggested that for surgical specimen cases with strong HER2 staining (membranous reactivity visible at $\times 2.5 / \times 5$ magnification) in $<10 \%$ of cells, retesting with fluorescence in situ hybridization or silver in situ hybridization may be warranted. If such a sample is fluorescence in situ hybridization- or silver in situ hybridizationpositive the tumor may be considered to be HER2positive similar to scoring on biopsy samples; scores for both assays should be indicated separately on the report. On the basis of current evidence, this staining pattern occurs in only a small percentage of cases but in the majority of cases that show small immunohistochemistry $3+$ tumor foci, the sample is also fluorescence in situ hybridization- or silver in situ hybridization-positive (Figures 2d-f).

Within the trastuzumab for GAstric cancer study it was not possible to determine a relationship between treatment benefit and degree of HER2-related tumor heterogeneity, mainly due to the small sample sizes of the respective subgroups involved. 

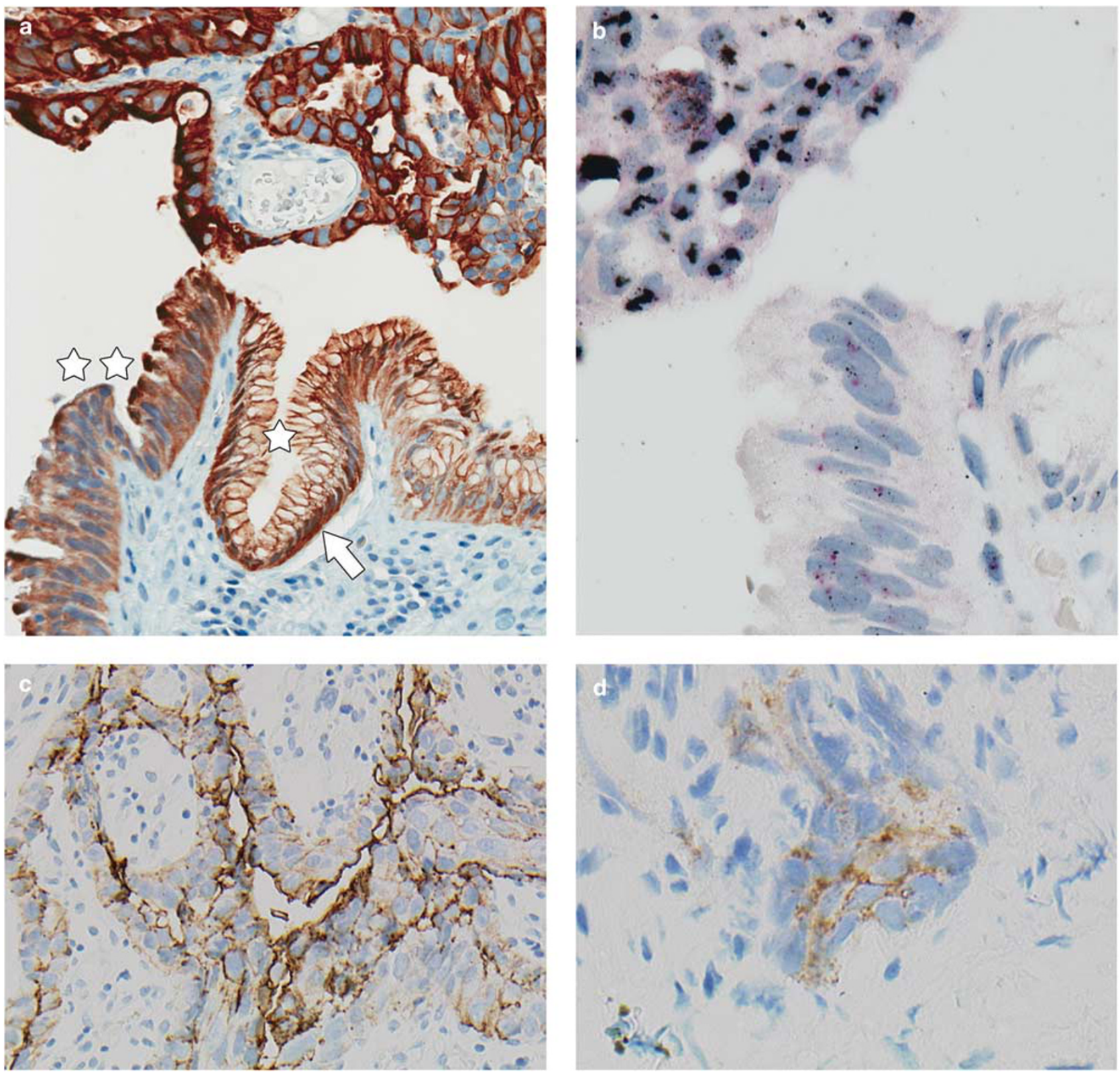

Figure 3 (a-d) Pitfalls in HER2 immunoscoring (4B5). (a) Biopsy specimen with poorly differentiated intestinal-type adenocarcinoma showing strong HER2 membranous staining (immunohistochemistry $3+$, upper half). Intestinal metaplasia (see star) and epithelial dysplasia (see double star) are seen showing a mixture of diffuse cytoplasmic staining with some nuclear (see arrow) and focally membranous staining of questionable specificity (lower half; $\times 20$ ). (b) Dual color bright-field in situ hybridization staining (same tumor sample as in Figure 1a) shows high amplification with clustering of HER2 gene signals in the cancerous region (upper half) but no HER2 gene amplification in either the metaplasia or the dysplastic epithelium (lower half; $\times 40$ ). (c-d) Staining artifacts that should be excluded from scoring and/or require in situ hybridization confirmation. (c) Strong pseudo-luminal staining due to shrinkage artifact $(\times 20)$ and $(\mathbf{d})$ granular (nonlinear) equivocal membranous staining in a group of tumor cells $(\times 40)$.

\section{Optimizing In Situ Hybridization}

As discussed previously, it is mandatory that samples classified as immunohistochemistry $2+$ should be retested by fluorescence in situ hybridization or silver in situ hybridization to assess the HER2 status of the tumor. As with immunohistochemistry, a standard operating procedure should be developed for fluorescence in situ hybridization and silver in situ hybridization within each testing laboratory, including standardization of protocols and laboratory equipment. It is important to note that samples may need to be treated differently according to whether fluorescence in situ hybridization or silver in situ hybridization will be utilized. In order to achieve a good in situ hybridization signal, it is important that sufficient well-preserved tumor tissue is collected and that samples are carefully 
prepared. Insufficient deparaffinization can lead to nuclear bubbles, and nuclear holes may be present as a result of overdigestion. ${ }^{28}$

It is strongly recommended that validated fluorescence in situ hybridization and silver in situ hybridization kits be used for testing in gastric cancer as per the manufacturer's instructions to ensure the production of accurate and reliable data. Preanalytical tissue preparation should also be performed according to the manufacturer's instructions for the in situ hybridization testing kits. Validated in situ hybridization testing kits that are commercially available are following: Ventana HER2 DNA probe (formerly known as BDISH; Roche Tissue Diagnostics), Ventana HER2 Dual ISH DNA Probe Cocktail (formerly known as DDISH; Roche Tissue Diagnostics), and the HER2 FISH pharmDx kit (Dako). Use of other in situ hybridization methodologies in gastric cancer (eg, PathVysion (Abbott), which is commonly used in countries where other tests are not yet approved, or chromogenic in situ hybridization) require validation of the assay against the validated tests.

Although both fluorescence in situ hybridization and silver in situ hybridization are approved by the European Medicines Agency for the retesting of immunohistochemistry $2+$ HER2 samples, it is widely accepted that silver in situ hybridization is a more suitable methodology than fluorescence in situ hybridization for gastric cancer, as it is a bright-field methodology and can therefore allow rapid identification of HER2-positive tumor foci within a heterogeneous sample. Concordance between the Ventana HER2 DNA Probe (formerly known as BDISH; (Roche Tissue Diagnostics)) and the HER2 FISH pharmDx kit (Dako) is high at $95.3 \%{ }^{35}$ and further concordance studies are warranted and encouraged. Silver in situ hybridization slides also have the advantage of being more suitable for long-term storage than fluorescence in situ hybridization slides, as there is no risk of bleaching.

Another key recommendation for HER2 testing is to align the use of immunohistochemistry and in situ hybridization (particularly silver in situ hybridization) in order to help identify heterogeneous areas of HER2 reactivity. Immunohistochemistry should be used as the initial test to identify HER2-positive regions, the location of which can then be used to guide the fluorescence in situ hybridization or silver in situ hybridization assessment (Figures $2 \mathrm{~b}$ and $\mathrm{c}$ ). The requirement for a bright-field methodology to identify heterogeneity further supports the use of immunohistochemistry as the initial HER2 testing modality in gastric cancer.

\section{Sample Analysis: In Situ Hybridization}

Fluorescence in situ hybridization and silver in situ hybridization results are expressed as the ratio between the number of copies of the HER2 gene and the number of copies of chromosome 17 within the nucleus counted in at least 20 cancer cells. The definition of fluorescence in situ hybridization or silver in situ hybridization positivity in gastric or gastro-esophageal junction cancer is a HER2: chromosome 17 ratio of $\geq 2.0$. For quality reasons, when using dual probes only cells with $\geq 1$ in situ hybridization signal for each probe should be assessed and cells with only one chromosome instead of the typical two are considered monosomic.

When analyzing in situ hybridization samples, it is important to consider how the area for testing should be assessed. The entire specimen should be screened for amplified regions at a $\times 20$ magnification; this is particularly important in fluorescence in situ hybridization samples where a bright-field image is not available. Areas with overlapping nuclei or high nonspecific background staining (dark field methods only) or where there is a weak signal or the presence of artifacts (eg, dust grains) should not be included in the interpretation. Using a $\times 40$ magnification, it is recommended that at least 20 evaluable, nonoverlapping cells are counted initially, including cells from different tumor areas to provide a more uniform score interpretation. In borderline amplification cases (ratio of 1.8-2.2) it is suggested to recount $\sim 20$ additional cells and consider counting cells in an alternative area of tissue or in another sample. If there is a cluster of, eg, only five HER2-amplified tumor cells, the final in situ hybridization result should still be based on a total of 20 counted cells. In cases such as these, there is a high probability that the HER2:chromosome 17 ratio will be $>2.0$.

In addition to the HER2:chromosome 17 ratio, the overall HER2 gene count is also an important consideration for scoring fluorescence in situ hybridization and silver in situ hybridization samples. If the ratio suggests borderline amplification (ratio close to 2.0), the total gene count should also be considered because a high gene count (ie, $>6$ copies) may indicate a HER2-positive score. As a consequence, and based on the expert opinion of the authors, it is recommended that if there are $>6$ copies of the HER2 gene seen with a single probe then the sample is considered positive. If there are four to six HER2 gene copies then a dual-probe test is advised and the ratio should be calculated by counting an additional 20 cells. If the number of HER2 gene copies is < 4, it is advised to score the tumors as HER2-negative.

It is also important to have a hematoxylin and eosin section of the tumor available as a guide to scoring the in situ hybridization slide. If a particularly low ratio is observed, it may be necessary to check for polysomy and re-check immunohistochemistry results (Figure 2g). Caution must be applied when interpreting polysomy in gastric cancer as sometimes this may represent a coamplification of the centromere region of chromosome 17 rather than a true polysomy (ie, increased copy 
number of the whole chromosome 17). According to the trastuzumab for GAstric cancer study data, polysomy (defined as $\geq 3$ chromosome 17 signals) occurred in only $4.1 \%$ of the entire screening population, inclusive of cases with amplification. ${ }^{22}$

\section{Quality Assurance}

In order to achieve quality HER2 testing results that are consistent across testing laboratories, it is strongly recommended that testing be performed by centralized reference laboratories wherever possible. In regions where centralized testing is not possible, testing should be conducted by experienced local pathology laboratories with previous experience of both immunohistochemical and in situ hybridization testing in breast cancer that have undergone comprehensive training specific to the analysis of gastric tumor samples. Centers should be responsible for performing adequate numbers of HER2 tests per site/year to maintain quality standards.

At the onset of diagnostic testing for HER2 in any centre, the initial 25-50 cases should always be analyzed in parallel using both immunohistochemistry and in situ hybridization. It is anticipated that the concordance rate in gastric cancer should be similar to breast cancer $(>90 \%)$ if the equivocal immunohistochemistry $2+$ group is disregarded for analysis purposes. Accordingly, in the trastuzumab for GAstric cancer trial the concordance rate between immunohistochemistry (HercepTest (Dako)) and fluorescence in situ hybridization (HER2 FISH pharmDx (Dako)), was $94.9 \%$ for immunohistochemistry $3+$ and in situ hybridization-positive cases (354/373 cases) and 92.5\% for immunohistochemistry $1+$ /immunohistochemistry 0 and in situ hybridization-negative cases (2519/2329 cases). ${ }^{22}$ In the aforementioned diagnostic series, concordance between immunohistochemistry (Ventana CONFIRM HER-2/neu (4B5) Rabbit Monoclonal Primary Antibody (Roche Tissue Diagnostics)) and silver in situ hybridization (Ventana HER2 Dual ISH DNA Probe Cocktail, formerly BDISH (Roche Tissue Diagnostics)), defined as immunohistochemistry $3+$ and silver in situ hybridization-positive or immunohistochemistry $1+$ / immunohistochemistry 0 and silver in situ hybridization-negative was $98 \%$ (103/105 cases; all immunohistochemistry $3+$ cases $(24 / 24)$ were also silver in situ hybridization-positive and 79/81 of the immunohistochemistry $1+/$ immunohistochemistry 0 cases were also silver in situ hybridization-negative). ${ }^{29}$ Though not currently used as standard practice, it is envisioned that partially automated sample scoring systems are a possibility for the future, and may also provide an objective measure of signal intensity. ${ }^{36}$

Analysis and scoring of HER2 testing results for gastric and gastro-esophageal junction cancer should be performed by a qualified, trained, board-certified pathologist, and technical staff should not be solely responsible for the analysis and/or interpretation of HER2 results. Owing to the differences between breast cancer and gastric cancer HER2 testing and scoring, it is imperative that personnel should be specifically trained in HER2 testing methods and interpretation for gastric cancer, irrespective of previous experience in breast cancer. This should include training on preanalytical procedures through to the scoring and interpretation of gastric cancer samples, as applying the breast cancer scoring system to gastric cancer samples may result in a large number of inaccurate HER2 scoring results that could lead to some patients with HER2-positive gastric cancer not being identified. It is anticipated that specific training and regular gastric cancerrelated teaching activities will increase the number of accurate HER2 testing results and ensure patients have access to suitable treatments.

All centers responsible for HER2 testing should be governed by a validated procedure, and subject to an internal quality assurance program. In addition to internal quality assurance programs, all technical and medical staff should undergo continual competency assessments and training. HER2 testing laboratories should actively participate in proficiency testing schemes though external quality assurance programs. Currently, quality assurance programs exist for HER2 testing in gastric cancer in the United Kingdom (United Kingdom National External Quality Assessment Service: http://www.ukneqas. org.uk), Nordic countries (Nordic Immunohistochemical Quality Control: http://www.nordiqc.org), Germany (Qualitätssicherungs-Initiative in der Pathologie Ringversuche: http://www.ringversuch.de), and France (Association Française D’Assurance Qualité en Anatomie et Cytologie Pathologiques: http://www. afaqap.org).

\section{Status of International Approvals}

Trastuzumab is approved for use in advanced/metastatic gastric or gastro-esophageal junction cancer in the European Union (by the European Medicines Agency), the United States (by the Food and Drug Administration), and in many other countries.

\section{Summary}

On the basis the data from the trastuzumab for GAstric cancer study, ${ }^{1}$ trastuzumab was approved by the European Medicines Agency for patients with metastatic gastric cancer. It is clear that accurate patient identification, and thus clinical benefit, is dependent on quality HER2 testing. The recommendations described here have been developed based on the trastuzumab for GAstric cancer study and the expert opinions of the authors who share a wealth of experience in HER2 testing. For an overview of the 
Table 3 Human epidermal growth factor receptor 2 (HER2) testing recommendations in gastric cancer, (a) immunohistochemistry and (b) in situ hybridization

(a) Immunohistochemistry

Testing recommendations

- Representative surgical samples or an adequate number of viable biopsy specimens (ideally six to eight) are required $\bigcirc$ If few biopsies are available, all viable specimens should be tested

- Immunohistochemistry should be the initial HER2 testing methodology for gastric cancer and bright-field methodologies are preferred wherever possible

HER2-positive per European Medicines Agency license: immunohistochemistry 3+ or immunohistochemistry 2+/fluorescence in situ hybridization-positive or immunohistochemistry $2+/$ silver in situ hybridization-positive

○ Borderline immunohistochemistry 1+/immunohistochemistry 2+ cases and samples with focal and intense membranous reactivity in $<10 \%$ cells may also be retested with fluorescence in situ hybridization or silver in situ hybridization (scores for both assays should be indicated separately on the report)

- Validated immunohistochemistry HER2 assays should be used

Scoring recommendations

- Due to the tumor heterogeneity (focal areas of positivity) and incomplete membrane staining commonly seen in gastric cancer, the gastric cancer-specific scoring criteria should be adhered to:

○ Surgical specimen cutoff: complete, basolateral, or lateral membranous reactivity in $\geq 10 \%$ of cells

Biopsy specimen cutoff: complete, basolateral, or lateral membranous reactivity in $\geq 5$ clustered cells

- The 'magnification rule' should be used in conjunction with the scoring criteria

- Borderline cases (immunohistochemistry 1+/immunohistochemistry $2+$ or focal staining in $<10 \%$ cells) that score fluorescence in situ hybridization-positive or silver in situ hybridization-positive may be considered HER2-positive (scores for both assays should be indicated separately on the report)

(b) In situ hybridization

Testing recommendations

- Tumor samples classified as immunohistochemistry 2+ should be retested by fluorescence in situ hybridization or silver in situ hybridization to assess HER2 status

- Silver in situ hybridization is a more suitable methodology than fluorescence in situ hybridization for assessing HER2 status in gastric tumor samples as it is a bright-field methodology and thus allows for rapid identification of HER2-positive tumor foci within a heterogeneous sample

- Validated in situ hybridization HER2 assays should be used

Scoring recommendations

- The definition of fluorescence in situ hybridization or silver in situ hybridization positivity in gastric or gastro-esophageal junction cancer is a HER2:chromosome 17 ratio of $\geq 2.0$

- The entire case should be screened for amplified regions (particularly important for fluorescence in situ hybridization samples where a bright-field image is not available)

- At least 20 evaluable, non-overlapping cells in the invasive component should be counted initially

- In borderline amplification cases, $\sim 20$ additional cells should be recounted or scoring should be performed in an alternative area of tissue

- The overall HER2 gene count is important:

$\bigcirc>6$ HER2 gene copies using single probe: considered positive

Four to six HER2 gene copies: dual probe test advised and the ratio should be recalculated by counting an additional 20 cells

Ensuring quality and timely HER2 testing results

- The use of validated immunohistochemistry and in situ hybridization tests is strongly recommended and appropriate controls should be included in each run

- Turnaround time from initial diagnosis to reporting of results should ideally not exceed 5 working days and a multidisciplinary approach is required

- Centralized testing is recommended wherever possible and all laboratories should participate in validated quality assurance programs

key recommendations for both immunohistochemistry and in situ hybridization, see Table 3.

Briefly, the main recommendations are that all patients with gastric cancer should be tested for HER2 status at the time of initial diagnosis, with biopsies being the preferred specimen type due to specimen quality reasons, and that testing and scoring should be performed with adherence to the recommendations specifically devised for gastric cancer. The subsequent treatment of patients with HER2-positive tumors will vary globally, dependent on local regulations and approvals, and as such the practical guidance provided here is intended to be broad and wide-reaching and should therefore be applicable across all regions following the European Medicines Agency approval. It is anticipated that as experience of HER2 testing in gastric cancer grows, these recommendations will continue to evolve.

\section{Disclosure/conflict of interest}

Josef Rüschoff has received honoraria for attendance at advisory boards from F Hoffmann-La Roche. 
HER2 testing within the trastuzumab for GAstric cancer trial was performed at Targos $\mathrm{GmbH}$ under his guidance and was reimbursed by F Hoffmann-La Roche. Giuseppe Viale has received honoraria and consultation fees from $F$ Hoffmann-La Roche and Dako. Michael Bilous has received sponsorship from F Hoffmann-La Roche for attending conferences and honoraria for attendance at advisory boards. Frédérique Penault-Llorca has received honoraria for attendance at advisory boards and research Grants from F Hoffmann-La Roche. Manfred Hofmann has received honoraria and consultation fees from $F$ Hoffmann-La Roche. Wedad Hanna has received honoraria to attend the advisory board and small grants for pilot studies from F Hoffmann-La Roche, and pilot study Grant support from Roche Canada. Marc van de Vijver has received honoraria, consultation fees, and research Grants from F Hoffmann-La Roche. Robert Y Osamura has received sponsorship from F Hoffmann-La Roche for attending conferences and honoraria for attendance at advisory boards.

\section{Acknowledgements}

Support for third-party writing assistance for this manuscript was provided by F Hoffmann-La Roche. This paper results from an advisory board meeting held in July 2010, which was supported by F Hoffmann-La Roche. Attendees received honoraria for attendance at the advisory board.

\section{References}

1 Bang YJ, Van Cutsem E, Feyereislova A, et al. Trastuzumab in combination with chemotherapy versus chemotherapy alone for treatment of HER2-positive advanced gastric or gastro-oesophageal junction cancer (ToGA): a phase 3, open-label, randomised controlled trial. Lancet 2010;376:687-697.

2 Bilous M, Dowsett M, Hanna W, et al. Current perspectives on HER2 testing: a review of national testing guidelines. Mod Pathol 2003;16:173-182.

3 Bilous M, Osamura RY, Rüschoff J, et al. HER-2 amplification is highly homogenous in gastric cancer. Hum Pathol 2010;41:304-305.

4 Dowsett M, Hanna WM, Kockx M, et al. Standardization of HER2 testing: results of an international proficiency-testing ring study. Mod Pathol 2007;20: $584-591$.

5 Hanna W, O'malley FP, Barnes P, et al. Updated recommendations from the Canadian National Consensus Meeting on HER2/neu testing in breast cancer. Curr Oncol 2007;14:149-153.

6 Hofmann M, Stoss O, Shi D, et al. HER2 status evaluation for gastric cancer: a consensus study. Gastrointestinal Cancers Symposium, Abstract 24 San Francisco, CA, USA; January, 2006, pp 26-28.

7 Hofmann M, Stoss O, Shi D, et al. Assessment of a HER2 scoring system for gastric cancer: results from a validation study. Histopathology 2008;52:797-805.
8 Garcia M, Jemal A, Ward EM, et al. Global Cancer Facts and Figures 2007. American Cancer Society 2007; Atlanta, GA, USA, 2010, Available at: http://www.cancer. org/acs/groups/content/@nho/documents/document/global factsandfigures2007rev2p.pdf (accessed 12 November 2010).

9 Kamangar F, Dores GM, Anderson WF. Patterns of cancer incidence, mortality, and prevalence across five continents: defining priorities to reduce cancer disparities in different geographic regions of the world. J Clin Oncol 2006;24:2137-2150.

10 Ferlay J, Shin HR, Bray F, et al. Estimates of worldwide burden of cancer in 2008: GLOBOCAN 2008. Int J Cancer 2010;127:2893-2917.

11 Inoue M, Tsugane S. Epidemiology of gastric cancer in Japan. Postgrad Med J 2005;81:419-424.

12 Lee KJ, Inoue M, Otani T, et al. Gastric cancer screening and subsequent risk of gastric cancer: a large-scale population-based cohort study, with a 13-year followup in Japan. Int J Cancer 2006;118:2315-2321.

13 Cunningham SC, Kamangar F, Kim MP, et al. Survival after gastric adenocarcinoma resection: eighteen-year experience at a single institution. J Gastrointest Surg 2005;9:718-725.

14 Horner MJ, Ries LAG, Krapcho M, et al. National Cancer Institute, Bethesda, MD, USA, based on November 2008 SEER data submission, posted to the SEER web sitehttp://seer.cancer.gov/csr/1975_2006/2009.

15 Slamon DJ, Godolphin W, Jones LA, et al. Studies of the HER-2/neu proto-oncogene in human breast and ovarian cancer. Science 1989;244:707-712.

16 Koeppen HK, Wright BD, Burt AD, et al. Overexpression of HER2/neu in solid tumours: an immunohistochemical survey. Histopathology 2001;38:96-104.

17 Slamon DJ, Leyland-Jones B, Shak S, et al. Use of chemotherapy plus a monoclonal antibody against HER2 for metastatic breast cancer that overexpresses HER2. N Engl J Med 2001;344:783-792.

18 Romond EH, Perez EA, Bryant J, et al. Trastuzumab plus adjuvant chemotherapy for operable HER2positive breast cancer. N Engl J Med 2005;353: 1673-1684.

19 Herceptin (trastuzumab) prescribing information. 2009.

20 National Comprehensive Cancer Network (NCCN). Clinical Practice Guidelines in Oncology: Breast Cancer V.1.2009. 2009.

21 Gravalos C, Jimeno A. HER2 in gastric cancer: a new prognostic factor and a novel therapeutic target. Ann Oncol 2008;19:1523-1529.

22 Chung HC, Bang YJ, Xu JM, et al. Human epidermal growth factor receptor 2 (HER2) in gastric cancer (GC): results of the ToGA trial screening programme and recommendations for HER2 testing. ECCO Abstract 6511, Vol. 34; Berlin, Germany, 2009.

23 Park DI, Yun JW, Park JH, et al. HER-2/neu amplification is an independent prognostic factor in gastric cancer. Dig Dis Sci 2006;51:1371-1379.

24 Tanner M, Hollmen M, Junttila TT, et al. Amplification of HER-2 in gastric carcinoma: association with Topoisomerase IIalpha gene amplification, intestinal type, poor prognosis and sensitivity to trastuzumab. Ann Oncol 2005;16:273-278.

25 Brien TP, Depowski PL, Sheehan CE, et al. Prognostic factors in gastric cancer. Mod Pathol 1998;11:870-877.

26 Takehana T, Kunitomo K, Kono K, et al. Status of c-erbB-2 in gastric adenocarcinoma: a comparative study of immunohistochemistry, fluorescence in situ 
hybridization and enzyme-linked immuno-sorbent assay. Int J Cancer 2002;98:833-837.

27 Herceptin EU SmPC. Available at: http://www.ema. europa.eu/docs/en_GB/document_library/EPAR - Product Information/human/000278/WC500074922.pdf 2010, (accessed 12 November 2010).

28 Rüschoff J, Nagelmeier I, Baretton G, et al. Her2 testing in gastric cancer. What is different in comparison to breast cancer? Pathologe 2010;31:208-217.

29 Rüschoff J, Dietel M, Baretton G, et al. HER2 diagnostics in gastric cancer-guideline validation and development of standardized immunohistochemical testing. Virchows Arch 2010;457:299-307.

30 Wolff AC, Hammond ME, Schwartz JN, et al. American Society of Clinical Oncology/College of American Pathologists guideline recommendations for human epidermal growth factor receptor 2 testing in breast cancer. J Clin Oncol 2007;25:118-145.

31 Heresbach D, Napoléon B, Delchier J-C, et al. Consensus en endoscopie digestive (CED): indications des biopsies digestives au cours de la fibroscopie ou du dépistage des néoplasies œeso-gastro-duodénales. Acta Endoscopia 2009;39:206-211.
32 Recommendations from the National French Group. http://www.tncd.org 2010, (accessed 12 November 2010). In French.

33 Middleton LP, Price KM, Puig P, et al. Implementation of American Society of Clinical Oncology/College of American Pathologists HER2 Guideline Recommendations in a tertiary care facility increases HER2 immunohistochemistry and fluorescence in situ hybridization concordance and decreases the number of inconclusive cases. Arch Pathol Lab Med 2009;133:775-780.

34 Bussolati G, Gugliotta P, Volante M, et al. Retrieved endogenous biotin: a novel marker and a potential pitfall in diagnostic immunohistochemistry. Histopathology 1997;31:400-407.

35 Powell WC, Zielinski D, Ranger-Moore J, et al. Determining the HER2 status in gastric cancer: a method comparison study of two patient cohorts. Gastrointestinal Cancers Symposium, Abstract 17, 2010, Orlando, FL, USA.

36 Dobson L, Conway C, Hanley A, et al. Image analysis as an adjunct to manual HER-2 immunohistochemical review: a diagnostic tool to standardize interpretation. Histopathology 2010;57:27-38. 